\section{Kinder mit leichtem Asthma}

\section{Cromone Mittel der Wahl}

\author{
Bei Kindern mit leichter Asthmasymptomatik sind Cromone wie \\ DNCG Mittel der ersten Wahl. Sie verfügen neben einer mastzellsta- \\ bilisierenden Wirkung auch über antientzündliche Effekte und sind \\ in ihrer klinischen Wirksamkeit gut dokumentiert. Wachstumsretar- \\ dierungen wurden unter dieser Dauermedikation nicht gesehen.
}

$\mathrm{D}$ as allergische Asthma bronchiale manifestiert sind bei rund $70 \%$ der Patienten mit einer leichten Symptomatik, bei Kindern dürfte dieser Prozentsatz noch höher liegen. Gerade bei diesen leichteren Formen ist bei der Auswahl der antientzündlichen Basismedikation eine sorgfältige Nutzen-RisikoAbwägung vorzunehmen. Grund genug, so D. Reinhardt (München), auch wieder einmal einen Blick auf die bewährte Cromoglicinsäure zu werfen.

\section{DNCG wirkt antiinflammatorisch}

Das Dinatriumsalz der Cromoglicinsäure (DNCG) wird in vielen Lehrbüchern noch als Mastzellstabilisator geführt. Doch neben diesem Eingriff in einen sicherlich zentralen Pathomechanismus verschiedener Allergieformen wirkt DNCG wie die Kortikoide antientzündlich - wenn auch nicht so stark, wie Reinhardt einschränkte.

Dazu gibt es eine Vielzahl von Belegen, sagte Reinhardt und zitierte als Beispiel eine bereits 1984 durchgeführte Untersuchung. Danach reduzierte eine DNCG-Therapie bei Asthmatikern die Zahl der Eosinophilen in der bronchoalveolären Lavage signifikant. Andere Untersuchungen bestätigten diese Ergebnisse: Nach 12-wöchiger DNCGGabe ging z.B. die Zahl von Eosinophilen als auch von Mastzellen signifikant zurück. Auch die Produktion verschiedener Interleukine nach Allergenkontakt verminderte sich unter DNCG.

Um die klinische Wirksamkeit von DNCG weiß jeder erfahrene Arzt. Um so erstaunter war man, als 1997 in der renommierten Fachzeitschrift Lancet eine Publikation von M. J. A. Tasche et al. erschien, die in der Feststellung mündete, bei Kindern sei „die Wirksamkeit von DNCG mit der von Plazebo vergleichbar."

\section{Klinische Wirksamkeit belegt}

Doch hatte die Studie methodische Mängel, insbesondere hinsichtlich der Definition des Asthma. Reinhardt: „Diese Publikation können Sie vergessen." Ihr stehen eine Reihe von Untersuchungen gegenüber, die bei Kindern mit leichtem bis mittelschwerem Asthma unter DNCG eine hochsignifikante Symptomreduktion auswiesen, vergleichbar mit der von Kortikoiden.

Auch beim Rückgang der bronchialen Hyperreagibilität konnte DNCG seine antiinflammatorische Potenz unter Beweis stellen - hier schnitten die Kortikoide allerdings besser ab.

Bewährt hat sich DNCG auch beim anstrengungsinduzierten Asthma, wobei durch die Dosiserhöhung von 1 auf $5 \mathrm{mg}$ mit keiner Wirkungssteigerung mehr zu rechnen ist, so das Ergebnis einer weiteren Studie.

\section{Keine Wachstumsretardierung}

„DNCG wirkt antiinflammatorisch und zeigt eine klinische Wirkung bei Kindern mit leichtem Asthma, die mit der von Kortikoiden in etwa vergleichbar ist", resümierte Reinhardt. Diese Erkenntnis erhält insofern besonderes $\mathrm{Ge}$ wicht, als dass auch unter den neueren topischen Kortikoiden bei Kindern mit genwachstums gerechnet werden muss. Die Schwellendosis liegt hier teilweise im Bereich der therapeutischen Dosis.

„Bei Kindern mit einem Asthma vom Schweregrad II werden also bei der Verordnung von Kortikoiden möglieiner negativen Beeinflussung des Län- cherweise unnötige Wachstumsretardierungen in Kauf genommen. Hier hat das etwas weniger stark wirksame DNCG durchaus seinen Stellenwert. Bei Asthma vom Schweregrad III und IV müssen natürlich Kortikoide gegeben werden", sagte Reinhardt.

Eine solche Stufentherapie deckt sich mit den Empfehlungen der Gesellschaft für pädiatrische Pneumologie. Danach gilt DNCG als Mittel der ersten Wahl für Kinder mit leichtem Asthma. Stellt sich nach sechswöchiger Monotherapie kein ausreichender Therapieeffekt ein, kann mit inhalativen Betaagonisten wie Reproterol (als Fixkombination z.B. in Aarane ${ }^{\circledR}$ ) kombiniert werden, so der Pädiater: „Reproterol ist mindestens genauso gut wirksam wie Salbutamol, es hat sogar eine höhere intrinsische Aktivität."

$b k$

\section{Reinhardt D}

1. Interdisziplinäres Aventis Allergie- \& Asthma-Forum 2000, Venedig, 2000.

Leukotrien-Antagonisten weitere Studien sind nötig

Der Stellenwert von Leukotrien-Rezeptorantagonisten wie Montelukast in der Behandlung von kindlichem Asthma lässt sich nach Reinhardt noch nicht abschätzen. In den offiziellen Stufenschemata der Fachgesellschaften tauchen sie bisher nur als Fußnote auf, erfreuen sich aber bei Ärzten und Kindern großer Beliebtheit.

Die bisher seit der Markteinführung vorgelegten 2 Studien seien aber für eine wissenschaftliche Beurteilung bei weitem nicht ausreichend. Reinhardt: „Es bleibt abzuwarten, ob wir weitere Studien zu sehen bekommen, die zeigen, wie sich dieses Therapieprinzip bewährt."

Solche Studien sind auch deshalb von Interesse, weil mit diesem theoretisch sicherlich guten Therapieansatz nur in einen einzigen Pathomechanismus beim Asthma bronchiale eingegriffen wird - möglicherweise ist das auf lange Sicht aber zu wenig. „Auch ist die Problematik der möglichen Entwicklung eines Churg-Strauss-Syndroms noch nicht endgültig vom Tisch", so Reinhardt. 